\title{
Design and Implementation of an Optimized Double Closed-loop Control System for MEMS Vibratory Gyroscope
}

\author{
Fang Chen, Weizheng Yuan, Honglong Chang, Guangmin Yuan, Jianbing Xie, Michael Kraft
}

\begin{abstract}
This work describes the development and experimental evaluation of a MEMS vibratory gyroscope using an optimized double closed-loop control strategy. An automatic gain control (AGC) self-oscillation interface is used to resonate the gyroscope in the drive mode; the sense mode is controlled by a sixth-order continuous-time, force-feedback band-pass sigma-delta modulator (BP- $\Sigma \Delta \mathrm{M})$. The parameters of both control loops were optimized by a genetic algorithm (GA). System level simulations show that the settling time of the drive mode self-oscillation is $125 \mathrm{~ms}$; the root-mean-square (RMS) displacement of the proof mass is less than $30 \mathrm{~nm}$ in the sense mode and the signal-to-noise ratio (SNR) is $90 \mathrm{~dB}$ in a bandwidth of $64 \mathrm{~Hz}$ with a $200 \%$ s angular rate input signal. The system was implemented using a symmetrical and fully decoupled silicon on insulator (SOI) gyroscope operating at atmospheric with the circuit implemented on printed circuit board (PCB). The measured power spectral density (PSD) of the output bitstream showed an obvious band-pass noise shaping and a deep notch at the gyroscope resonant frequency. The measured noise floor was approximately $-120 \mathrm{dBV} / \mathrm{Hz}^{1 / 2}$. In the drive mode, the relative drift of the resonant frequency and amplitude was $3.2 \mathrm{ppm}$ and 10.7ppm for 1 hour measurements, respectively. The settling time, scale factor, zero bias stability and bandwidth of the gyroscope controlled by the optimized control system were $200 \mathrm{~ms}, 22.5$ $\mathrm{mV} / \% / \mathrm{s}, 34 \% \mathrm{~h}$ and $110 \mathrm{~Hz}$, respectively. This compared to a non-optimized system for which the corresponding values were $300 \mathrm{~ms}, 17.3 \mathrm{mV} / \% / \mathrm{s}, 58^{\circ} / \mathrm{h}$ and $98 \mathrm{~Hz}$, hence by GA optimization a considerable performance improvement was achieved.
\end{abstract}

Index Terms-MEMS, gyroscope, AGC, BP- $\Sigma \Delta \mathrm{M}$, double closed-loop, genetic algorithm (GA)

Manuscript received January 17, 2013. This work was supported by the Chinese New Century Excellent Talents in University (Grant no NCET-10-0077), Chinese National Science Foundation (Grant no 61273052), Shaanxi Province Science and Technology Planning and Innovative project (Grant no 2011KTCQ01-26).

F. Chen, W. Yuan, H. Chang, G. Yuan, J. Xie are with the Key Laboratory of Micro/Nano Systems for Aerospace, Northwestern Polytechnical University, Ministry of Education, Xi'an 710072, China (e-mail: fchen1983@mail.nwpu.edu.cn; yuanwz@nwpu.edu.cn; changhl@nwpu.edu.cn; yuangm@nwpu.edu.cn; xiejb@nwpu.edu.cn).

M. Kraft is with the Faculty of Engineering Sciences, University of Duisburg-Essen, D-57057, Germany (e-mail: michael.kraft@uni-due.de).

Copyright (c) 2012 IEEE. Personal use of this material is permitted. However, permission to use this material for any other purposes must be obtained from the IEEE by sending a request to pubs-permissions@ieee.org.

\section{INTRODUCTION}

$\mathrm{M}$ ICRO-electro-mechanical-system (MEMS) vibratory gyroscopes have attracted tremendous attention in recent years due to their booming market demands, especially in the automotive and consumer electronics industry, and for navigation systems [1-4]. The principle of operation of a MEMS vibratory gyroscope is based on the Coriolis force [2, 3]. There are two orthogonal vibration modes: drive and sense mode, with two corresponding control loops for each mode. The Coriolis force transfers energy from the drive to the sense mode of a two degree of freedom resonant mechanical structure. The design of a gyroscope therefore needs to address both control loops; their joined optimization is crucial for the overall performance of the sensor. This paper presents a new approach toward a unified design strategy for both control loops.

Since the Coriolis force is proportional to the velocity of the proof mass along the driven axis, it is fundamental to maintain a constant drive velocity under practical variation of mechanical parameters. A commonly used approach is to use an automatic gain control (AGC). AGC is a control strategy that employs a nonlinear function to minimize the difference between the actual signal and a desired value [5]. Many research groups [5-13] have adopted an AGC circuit for a closed-loop drive control system of a gyroscope to initiate an oscillation with constant amplitude at the resonant frequency, however the optimization remains challenging. A proportional-integral (PI) controller is the prevailing method for the amplitude regulation because of its zero steady-state error; however, this leads to a trade-off problem between stability and settling time [6]. In [7], a digital AGC and PI controller with switched capacitor technology was used, but the control algorithm was investigated only in simulations and not evaluated in a hardware implementation. The design in [8] proposed another scheme of AGC with a PI controller to obtain self-oscillation; it decouples the angle and gain of the self-oscillation, therefore these parameters can be optimized separately. However, the optimization method again was not verified in hardware. The work presented in [9-11] described a detailed theoretical analysis of a nonlinear control system (AGC) for the JPL-Boeing gyroscope [12] and is based on an 
averaging method. The design presented in [13] applied the Lyapunov criterion to guarantee the stability of the AGC loop which requires an appropriately chosen Lyapunov candidate function. The study in [5] also employed an averaging and linearization method to establish a nonlinear mathematical model for the AGC and PI control system, which was evaluated through numerical simulation. However, the process was time-consuming and mathematically challenging. Eminoglu et al [6] proposed an optimized analytical design procedure for the AGC and PI analog controllers. This design procedure can obtain very fast starting MEMS vibratory gyroscope, however, it is only suitable for gyroscope packaged under high-vacuum.

Embedding the sense mode of a MEMS vibratory gyroscope in a high-order sigma-delta modulator $(\Sigma \Delta \mathrm{M})$ has proven to be advantageous, in terms of increased zero bias stability, linearity and direct digital output [14-22]. The control loop in [14, 15] used a fourth-order low-pass $\Sigma \Delta \mathrm{M}$; two electronic integrators were cascaded with the micromachined sensing element. Raman et al [16, 17] described a digitally controlled MEMS gyroscope with unconstrained sigma-delta force-feedback architecture, achieving a resolution of $0.025 \% / \mathrm{s} / \mathrm{Hz}^{1 / 2}$. The company Imego $\mathrm{AB}$ reported a fifth-order low-pass $\Sigma \Delta \mathrm{M}$ interface for a MEMS gyroscope operating in vacuum, with an Allan variance stability of $3.2^{\circ} / \mathrm{h}$ [18]. These aforementioned architectures are all principally low-pass electromechanical $\Sigma \Delta \mathrm{M}$, which required a relatively high sampling frequency compared to the bandwidth of the gyroscope. Therefore, as reported in [19-21], the sense mode of a MEMS vibratory gyroscope lends itself to being incorporated in a band-pass $\Sigma \Delta \mathrm{M}$ force feedback control loop. The electronic integrators of a low pass modulator are replaced with electronic resonators. Complex pairs of zeros are generated in the quantization noise transfer function $(Q N T F)$ of the closed loop system, which produce notches in the frequency response further suppressing the total in-band quantization noise. Consequently, the sampling frequency of the band-pass $\Sigma \Delta \mathrm{M}$ control system can be greatly decreased. A lower sampling frequency can also considerably reduce the power dissipation of the interface electronics. The work in [22] implemented a sixth-order band-pass continuous-time $\Sigma \Delta \mathrm{M}$ interface for a MEMS gyroscope as a PCB circuit. Preliminary measurements showed that the power spectral density (PSD) of the output bitstream achieved a noise floor of $-90 \mathrm{dBV} / \mathrm{Hz}^{1 / 2}$. However, no further characteristics were reported, in particular the performance of gyroscope band-pass $\Sigma \Delta \mathrm{M}$ was not tested in presence of an angular rate input signal.

The design of a double closed-loop MEMS gyroscope interface is extremely complex as the control system is highly nonlinear, and a multiple parameter optimization problem. Work to date mainly makes use of the conventional design approach which is to linearize the drive and sense control loops enabling the use of standard control theory. For example, in an AGC control loop, an averaging method is applied to generate an approximate linear model [5]; in the $\Sigma \Delta \mathrm{M}$ control loop, the 1-bit quantiser is replaced with a gain and a white quantization noise source [19, 20], then the optimization methodology is based on root locus techniques [21] or directly on the transfer function [23]. These linear methodologies neglect all second order effects and nonlinearities in the system, however these can be of importance for AGC and $\Sigma \Delta \mathrm{M}$ control loops. In [24] a new approach was used which is based on optimizing the parameters of a band-pass $\Sigma \Delta \mathrm{M}$ for the gyroscope sense mode by a Genetic Algorithm (GA), but the drive mode control system was not described.

Work presented in this paper extends the GA optimization to the entire gyroscope sensor system. First, it describes the design of a double closed-loop control system for a MEMS vibratory gyroscope operating at atmospheric pressure consisting of: i) an automatic gain control (AGC) circuit without a PI to realize the self-oscillation in the drive mode; and ii) the sense mode embedded in a sixth-order continuous-time band-pass sigma-delta modulator (BP- $\Sigma \Delta \mathrm{M})$ force feedback control loop. Thereafter, a comprehensive optimization methodology based on a GA and Monte Carlo simulations, both using non-linear system level models, was applied to the implementation of the double closed-loop interface. This cannot only reduce the settling time of the self-oscillation in the drive mode (which is an important parameter for the start-up time of a gyroscope), but also improve the signal to noise ratio (SNR) of the gyroscope. Finally, in order to demonstrate the validity of the approach, the optimized double closed-loop interface was implemented using off-the-shelf electronic components on a PCB (printed circuit board). The gyroscope sensing element used in this work was fabricated in a SOI (silicon on insulator) process and is described in $[25,26]$. The experimental test results verified the functionality of the optimized MEMS vibratory gyroscope double closed-loop control system.

\section{DOUBLE CLOSED-LOOP CONTROL SYSTEM}

\section{A. Design and linear analytical considerations}

The first step of the design methodology is to choose an appropriate system architecture. An initial noise shaping performance analysis can be done by using a linearized model. Here, a block diagram of the proposed double closed-loop control system in form of a Matlab/Simulink model is shown in Fig. 1. The parameters of the MEMS gyroscope used for design and simulation are listed in Table I. Fig. 1(a) depicts the block diagram of the AGC drive mode control system to stimulate self-oscillation. It can not only drive the proof mass to vibrate at constant amplitude but also self-adjust effectively in the presence of external disturbances. The displacement of the drive comb fingers $x(t)$ is converted to a voltage through a capacitive readout circuit with conversion gain $K_{p o}$ and a boost amplifier with a gain factor of $K_{b s t}$. A low pass filer $H_{L}(s)$ is used to eliminate high-frequency components from the capacitive read-out circuit. A phase shifter $H_{P}(s)$ is used to compensate phase delay in the AGC loop; the electrostatic 


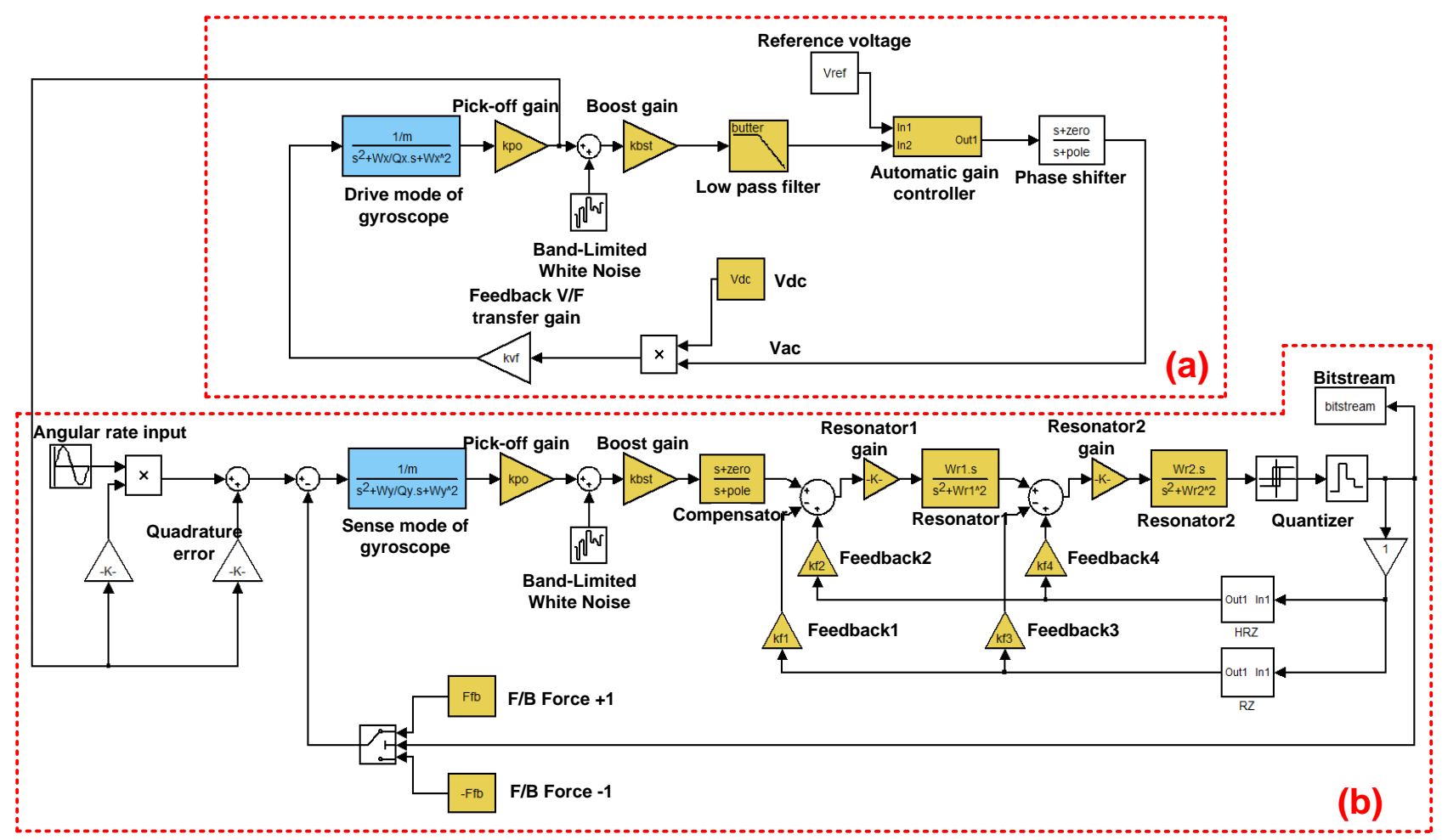

Fig. 1. Simulink model of the proposed double closed-loop control system for MEMS gyroscope. (a) AGC self-oscillation control loop for the drive mode (b) sixth-order band-pass electromechanical $\Sigma \Delta \mathrm{M}$ interface for the sense mode. parameters that are changed by the GA are highlighted in yellow.

feedback force $F_{v f}(t)$ is applied to the gyroscope as the excitation signal. An automatic gain control (AGC) is used to adjust the amplitude of the vibration to a target reference value. Fig. 1(b) depicts the proposed single loop sixth-order continuous-time BP- $\Sigma \Delta \mathrm{M}$ control system for the sense mode, which consists of a capacitive readout circuit with gain $K_{p o}^{\prime}$, an boost amplification circuit with gain $K_{b s t}^{\prime}$, a compensator $C_{P}(s)$, two electronic resonators and a one-bit quantizer. The sensing element is cascaded by two electronic resonators with transfer functions $R_{1}(s)$ and $R_{2}(s)$; this considerably improves the quantization noise shaping [20]. The forward path of the control system introduces some phase delay which is significant for loop stability $[19,20]$; therefore, the compensator $C_{P}(s)$ is required to provide phase lead to ensure loop stability. A clocked one-bit quantizer is used to produce a digital bitstream as the output signal. This also controls the sign of the feedback electrostatic force on the proof mass to counteract the Coriolis force maintaining the proof mass close to its rest position. There are two different digital to analogue converters (DAC) required in the local feedback paths to each electronic resonator; a half-return-zero (HRZ) DAC and a return-zero (RZ) DAC. Each have separately tunable gain constants $K_{h r z}$ and $K_{r z}$ which are used to provide multi-feedback waveforms to retain the same frequency response as a discrete-time (DT) $\Sigma \Delta \mathrm{M}$ [27]. The double closed-loop control system parameters that are to be optimized by the GA are highlighted in yellow in fig. 1. There are three noise sources in this system: mechanical (Brownian) noise, electronic noise introduced by the circuit and quantization noise due to the analog to digital conversion process. As the front-end circuit is the dominant electronic source [19], an input-referred electronic white noise source with a value of $10 \mathrm{nV} / \mathrm{Hz}^{1 / 2}$ is included in the model; mechanical noise is neglected.

TABLE I

PARAMETERS OF THE GYROSCOPE USED FOR SIMULATION

\begin{tabular}{ccc}
\hline \hline \multicolumn{1}{c}{ Parameter } & Drive mode & Sense mode \\
\hline Mass of proof mass & $0.21 \mu \mathrm{g}$ & $0.21 \mu \mathrm{g}$ \\
Quality factor & 119 & 114 \\
Resonance frequency & $4.304 \mathrm{kHz}$ & $4.338 \mathrm{kHz}$ \\
Structural layer thickness & $28 \mu \mathrm{m}$ & $28 \mu \mathrm{m}$ \\
Comb finger length & $15 \mu \mathrm{m}$ & $15 \mu \mathrm{m}$ \\
Comb finger width & $4 \mu \mathrm{m}$ & $4 \mu \mathrm{m}$ \\
Nominal gap of the comb finger & $3 \mu \mathrm{m}$ & $3 \mu \mathrm{m}$ \\
Length of comb fingers (overlapping) & $5 \mu \mathrm{m}$ & $5 \mu \mathrm{m}$ \\
Number of sensing comb fingers & 396 & 396 \\
Number of feedback comb fingers & 636 & 636 \\
\hline \hline
\end{tabular}

For the AGC self-oscillation in the drive mode, the displacement $x(t)$ of the proof mass can be controlled by the AC component of the excitation signal, the self-oscillation frequency $\omega_{0}$ can track the resonant frequency of the drive mode $\left(\omega_{x}\right)$. Neglecting nonlinear effects, the governing linearized equations of the AGC control loop are as follows [5, 9, 10]:

$$
\begin{aligned}
& \frac{d^{2} x(t)}{d t^{2}}+\frac{\omega_{x}}{Q_{x}} \frac{d x(t)}{d t}+\omega_{x}^{2} x(t)=\frac{F_{v f}}{m_{x}} \\
& F_{v f}=K_{p o} K_{b s t} K_{l} K_{v f} G \frac{d x(t)}{d t}
\end{aligned}
$$

With $G$ defined as

$$
G=K_{v a r} K_{p}\left(V_{\text {ref }}-A\right)
$$


where $x(t)$ is the displacement of the proof mass; $A$ is the amplitude of $x(t), \omega_{x}$ and $Q_{x}$ are the resonant frequency and quality factor of the drive mode, $m_{x}$ is the effective mass of the drive mode; $F_{v f}$ is the electrostatic driving force; $K_{l}$ is the gain of the low pass filter, $V_{\text {ref }}$ is the reference control voltage, $K_{v a r}$ and $K_{p}$ are the gain of the AGC and phase shifter, respectively. From (1) and (2), the dynamic vibration equation can be rewritten as follows:

$$
\frac{d^{2} x(t)}{d t^{2}}+\left(\frac{\omega_{x}}{Q_{x}}-\frac{K_{p o} K_{b s t} K_{l} K_{v f} G}{m_{x}}\right) \frac{d x(t)}{d t}+\omega_{x}^{2} x(t)=0
$$

The aim of the gyroscope drive mode control system is to make the system damping approximately zero, Equation (4) indicates that the AGC control system allows the gyroscope to self-oscillate by dynamically adjusting the system damping. The condition for self-oscillation therefore is

$$
\omega_{x} m_{x}=Q_{x} K_{p o} K_{b s t} K_{l} K_{v f} G
$$

If the measured amplitude is larger than the desired reference value, the AGC will decrease $K_{\text {var }}$ to enlarge the system damping; conversely, if the oscillation amplitude is smaller than the reference, the AGC will increase $K_{v a r}$ to enlarge the resonant amplitude. The gyroscope adjusts itself until the damping of the system is equal to zero and the system will maintain constant amplitude; thus

$$
\frac{d^{2} x(t)}{d t^{2}}+\omega_{x}^{2} x(t)=0
$$

Equation (6) is a linear differential equation with the analytic solution

$$
x(t)=A \cos \left(\omega_{x} t+\theta_{x}\right)
$$

The phase angle condition for self-oscillation of the gyroscope sensing element is: $\theta_{s}+\theta_{x}+\theta_{r}=2 \mathrm{n} \pi$ (where $\mathrm{n}$ is an integer) [8], where $\theta_{s}$ is the phase shift introduced by the phase shifter $H_{P}(s), \theta_{r}$ is the phase shift of the other electronic components in the drive mode control circuit and $\theta_{x}$ is the phase shift introduced by the sensing element in the drive mode which is $-\pi / 2$ when oscillating at resonance. At resonance the proof mass displacement is maximized, therefore the driving force should be in phase with the velocity of the proof mass [5, 8]. Consequently, the control loop design needs to ensure that the total phase angle of the drive control loop is $\theta_{s}+\theta_{x}+\theta_{r}=-2 \pi$ (n being equal to -1 here).

For the sixth-order continuous-time BP- $\Sigma \Delta \mathrm{M}$ interface for the sense mode, the signal transfer function $(S T F)$, electronic noise transfer function $(E N T F)$ and quantization noise transfer function $(Q N T F)$ of the system can be derived as [19, 20,21]:

$$
\begin{gathered}
S T F(s)=\frac{2 m \omega_{x} M(s) C_{p}(s) R_{1}(s) R_{2}(s) K_{b s}^{\prime} K_{p o}^{\prime} K_{q}}{1+L(s)} \\
\operatorname{ENTF}(s)=\frac{C_{p}(s) R_{1}(s) R_{2}(s) K_{b s t}^{\prime} K_{q}}{1+L(s)} \\
Q N T F(s)=\frac{1}{1+L(s)}
\end{gathered}
$$

$$
\begin{aligned}
& L(s)=K_{q}\left[R_{2}(s)\left(K_{f 3}-K_{f 4}\right)+\prod_{i=1}^{2} R_{i}(s)\left(K_{f 1}-K_{f 2}\right)\right. \\
& \left.+M(s) C_{p}(s) \prod_{i=1}^{2} R_{i}(s) K_{b s t}^{\prime} K_{p o}^{\prime}\right]
\end{aligned}
$$

$R_{1}(s)$ and $R_{2}(s)$ is the transfer function of two electronic resonators:

$$
R_{1}(s)=R_{2}(s)=K_{r e s} \frac{s \omega_{y}}{s^{2}+\omega_{v}^{2}}
$$

To enable a linear analysis, the quantizer is modeled by a variable gain $K_{q}$ with additive white quantization noise. $K_{p o}^{\prime}$ is the gain of the preamplifier representing the conversion from proof mass displacement to voltage at the output of the capacitive measurement interface, $K_{b s t}^{\prime}$ is a boost gain. $K_{f i}$, $(i=1 . .4)$ represents the local feedback loop gains for high-return-to-zero (HRZ) and return-to-zero (RZ) DACs, respectively, and $M(s)$ is the sense mode transfer function of the gyroscope sensing element:

$$
M(s)=\frac{1}{m_{y}\left(s^{2}+\frac{\omega_{y}}{Q_{y}} s+\omega_{y}^{2}\right)}
$$

where $m_{y}$ is the effective mass of the sense mode, $\omega_{y}$ and $Q_{y}$ are the resonant frequency and quality factor of sense mode, respectively.

\section{B. Nonlinear system optimization and simulation}

Linearized analytical models are of limited use in predicting the performance and stability of a double closed-loop interface with the chosen control strategy. For example, in the sense mode high performance noise shaping requires a high overall loop order for which stability becomes a greater concern, and a simple linear model of the quantizer is a less reasonable assumption [28]. Furthermore, there is a non-linear term due to the dependence of the feedback force on the proof mass position which has serious implications on stability and performance and cannot predicted with a linear model [29]. Therefore, a genetic algorithm (GA) together with a parameterized system level model is suitable to be used to perform a multi-objective optimization [24, 30]. Here, we choose the following parameters as optimization goal functions: i) the proof mass displacement in the sense mode (which should be minimized) in the presence of an input angular rate signal with $200^{\circ} / \mathrm{s}$ amplitude at $32 \mathrm{~Hz}$, ii) the SNR of the output bitstream (which should be maximized), iii) the settling time of the self-oscillation in the drive mode (which should be minimized), iv) $\theta_{r}$ which should be within the range $[-1.5 \pi,-0.25 \pi]$ to ensure stability of the AGC, as the capacitive pick-off circuit and low pass filter lead to considerable phase shift. The proposed methodology is represented by the flow-chart depicted in fig. 2 [30].

Where 


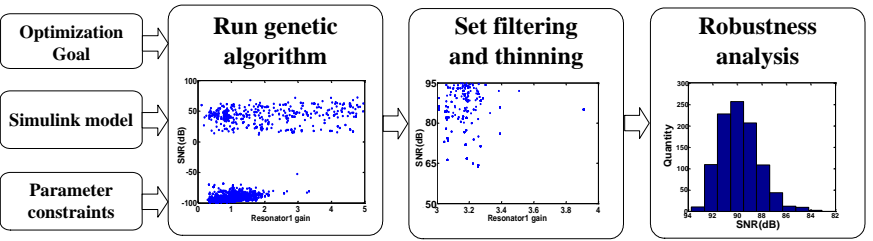

Fig. 2 Generic process flow of the proposed design algorithm [30]

The system parameters that are to be optimized (highlighted in yellow in fig. 1) form a parameter set termed an individual. An initial set of 100 individuals (termed a population) is randomly generated, within user specified constraints; they form the $1^{\text {st }}$ generation. The system then runs simulations (one for each individual) and records the performance objectives for each individual. Once the first generation has been simulated, the result is stored. The GA sort the results and then performs a number of post-simulation steps including picking the best individuals (elite preservation), generating a new random individuals (mutation) and cross fertilizing good individuals to create new offspring [30]. These steps form the second generation, which again consists of 100 individuals. In this work, any individuals for which the simulation yields an SNR below $60 \mathrm{~dB}$, root mean square (RMS) displacement more than $30 \mathrm{~nm}$, a settling time larger than $180 \mathrm{~ms}$ and $\theta_{r}$ not within the specified range are eliminated. The whole process continues until either a specified maximum number of generations has been reached (here, we used 100 generations) or it becomes obvious that a performance improvement is not apparent from generation to generation. The GA records the simulation results of all individual; for our example these are depicted as scatter plots in fig. 3 and 4: fig. 3 shows the SNR of all individuals as a function of one system parameter (the resonator 1 gain $K_{\text {res }}$; for the other parameters similar plots can be generated). Two clusters are visible: a SNR above zero indicates a stable system and an acceptable individual; a negative SNR implies instability and an unacceptable individual. The next step is a filtering and thinning algorithm which keeps a set of individuals with best performance and that are evenly distributed over the design space; this is depicted in fig. 4. Lastly, a robustness analysis based on Monte Carlo simulations is performed on these individuals to investigate control system stability in the presence of inevitable random parameter variations. Here, we ran 200 Monte Carlo simulations for each of the filtered and thinned individuals to reflect a statistically realistic variation. For choosing a final individual, a trade-off between performance and stability of the control system in the presence of parameter uncertainties has to be made by the designer. As a final step a comprehensive Monte Carlo simulation can be run on the chosen individual to obtain a statistically more significant variation analysis. Fig. 5 shows a histogram of 2000 Monte Carlo simulations of the individual that was selected to be taken forward for hardware implementation. The mean value of SNR in the double-closed loop system was $90 \mathrm{~dB}$ with a standard deviation 1.52. Finally, this individual is taken forward for hardware implementation. It is regarded as the optimal parameter set for the double closed-loop control system.

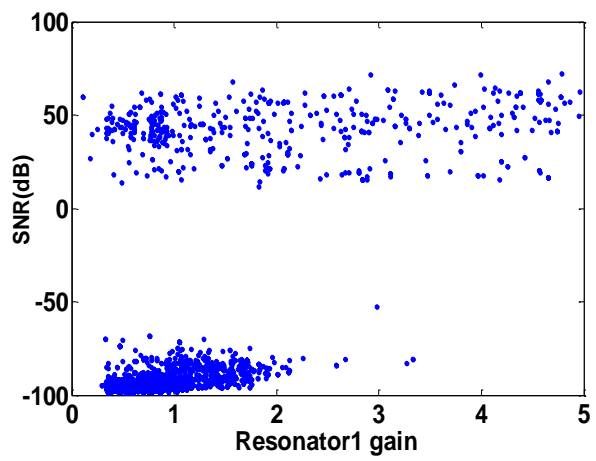

Fig. 3. SNR of the double closed-loop control system as a function of one system parameter (resonator gain $R_{1}(s)$ of the $\Sigma \Delta \mathrm{M}$ loop). A positive SNR indicates an individual that passed the goal function, and is preserved. On the contrast, an individual with negative SNR is eliminated.

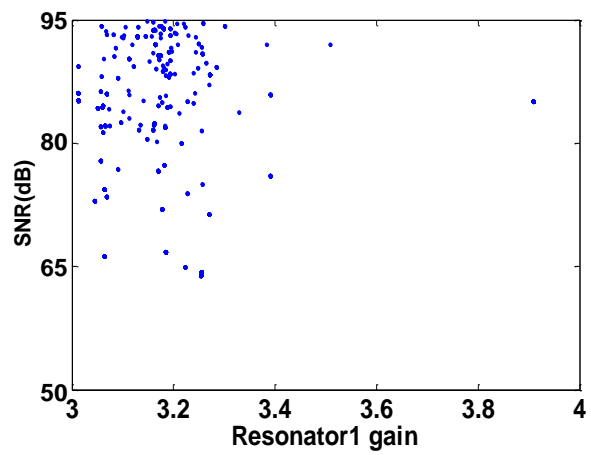

Fig. 4. SNR of the double closed-loop control system as a function of one system parameter (resonator gain $R_{1}(s)$ of the $\Sigma \Delta \mathrm{M}$ loop) after a filtering and thinning algorithm.

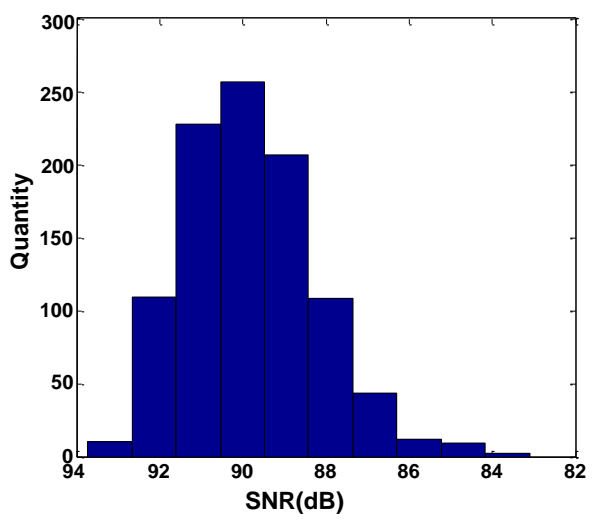

Fig. 5. Histogram showing the SNR of 2000 Monte Carlo simulations of the individual selected for hardware implementation

The parameters of the optimized design for the AGC drive mode control loop and sixth-order continuous-time BP- $\Sigma \Delta \mathrm{M}$ sense mode control loop are listed in table II and III, respectively. Fig. 6 shows that the simulated settling time of the self-oscillation drive loop for different drive loop gains: (a) loop gain less than the optimized value; (b) loop gain larger than the optimized value; (c) loop gain is equal to the optimized value. As can be seen, the settling time can be reduced with higher loop-gain. However, Overly-large gain will lead to overshoot and extend the settling time. The GA and Monte Carlo simulations were used to find the optimal value for this 
trade-off. The settling time of optimized loop is approximately $125 \mathrm{~ms}$; the drive mode displacement of the proof mass in steady state is $2.8 \mu \mathrm{m}$. Fig. 7 shows the relationship between $\left(\omega_{0} / \omega_{\mathrm{x}}\right)$ and $\theta_{s}$ for different quality factors, where $\omega_{0}$ is self-oscillation frequency, $\omega_{\mathrm{x}}$ is natural resonant frequency in drive mode. It can be seen that the small relative frequency difference can be realize by regulate $\theta_{s}$ close to $-0.5 \pi$.

TABLE II

OPTIMAL PARAMETERS FOR THE DRIVE MODE CONTROL LOOP

\begin{tabular}{ccc}
\hline \hline Parameter & Value & Unit \\
\hline Pick-off gain $\left(K_{p o}\right)$ & $1 \times 10^{6}$ & - \\
Boost gain $\left(K_{b s t}\right)$ & 150 & - \\
Low pass filter $H_{L}(\mathrm{~s})$ gain & 4.3 & - \\
AGC gain & 3.4 & - \\
Vdc & 5 & Volt \\
Vac (peak to peak) & 8.2 & Volt \\
$\theta_{s}$ & $-0.5 \pi$ & - \\
$\theta_{r}$ & $-1.0 \pi$ & - \\
\hline \hline
\end{tabular}

TABLE III

OPTIMAL PARAMETERS FOR THE SENSE MODE CONTROL LOOP

\begin{tabular}{ccc}
\hline \hline Parameter & Value & Unit \\
\hline Pick-off gain $\left(K_{p o}^{\prime}\right)$ & $1 \times 10^{6}$ & - \\
Boost gain $\left(K_{b s t}^{\prime}\right)$ & 649 & - \\
Compensator zero frequency & 4 & $\mathrm{kHz}$ \\
Compensator pole frequency & 194.17 & $\mathrm{kHz}$ \\
Local feedback loop gain $K_{f 1}$ & 2.56 & - \\
Local feedback loop gain $K_{f 2}$ & 1.35 & - \\
Local feedback loop gain $K_{f 3}$ & 3.72 & - \\
Local feedback loop gain $K_{f 4}$ & 1.07 & - \\
Resonator1 gain $K_{r e s}$ & 3.202 & - \\
Resonator2 gain $K_{r e s}$ & 3.202 & \\
Feedback voltage $V_{f}$ & 4.3 & Volt \\
\hline \hline
\end{tabular}

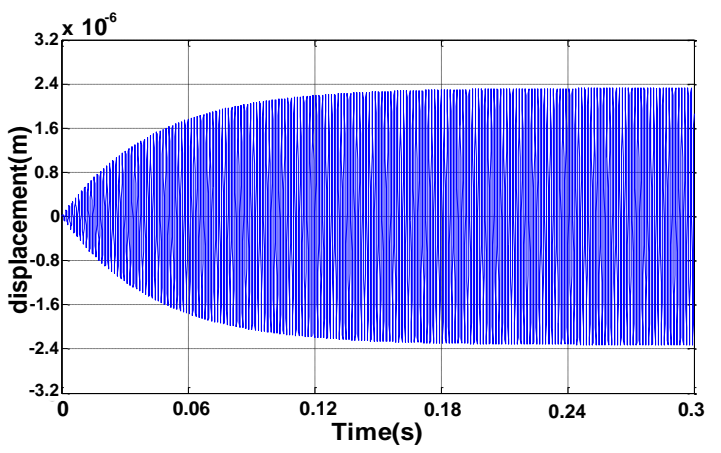

(a)

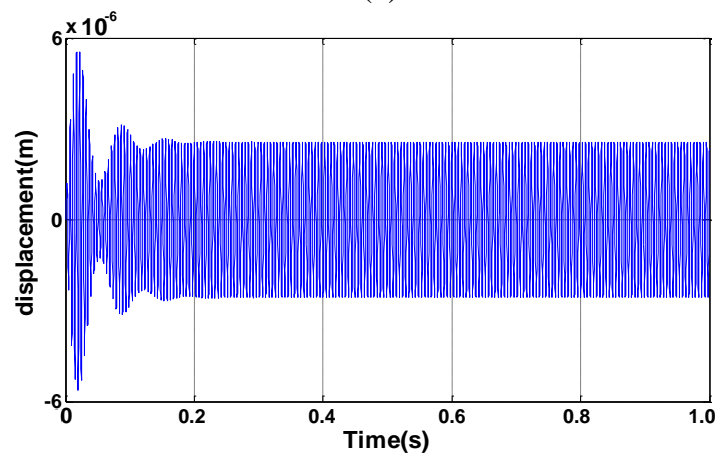

(b)

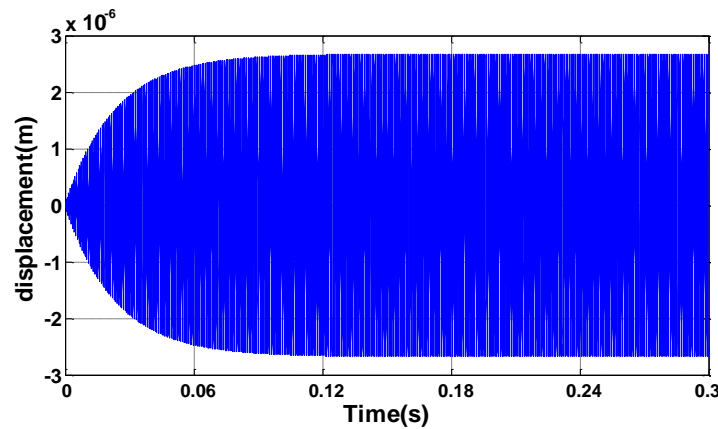

(c)

Fig. 6. Simulation result of the setting time of the self-oscillation in different drive loop gain: (a) loop gain less than the optimized value; (b) loop gain larger than the optimized value; (c) loop gain is equal the optimized value.

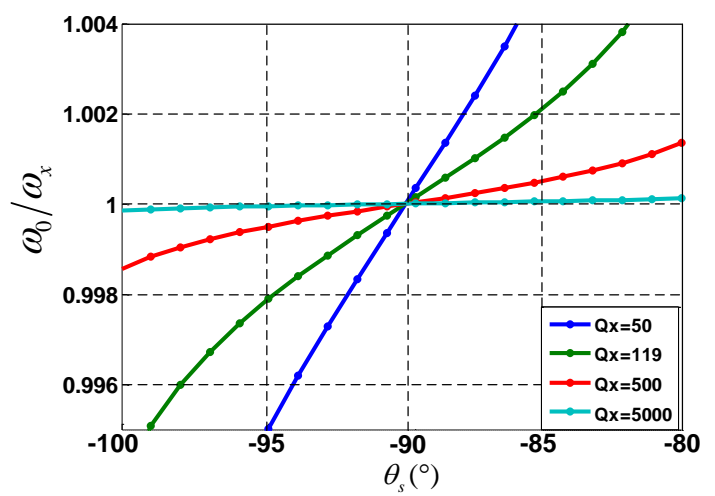

Fig. 7. The relationship between $\omega_{0} / \omega_{\mathrm{x}}$ and phase shift $\theta_{s}$ introduced by the phase shifter $H_{P}(s)$ in different quality factor.

For the parameters of the optimized design (table III) for the sixth-order continuous-time BP- $\Sigma \Delta \mathrm{M}$ sense mode control loop, the transfer functions (STF, QNTF and ENTF) can be calculated using equations (8)-(10); they are shown as magnitude plots in fig. 8. A notch is created in the signal band to suppress the quantization noise. For the signal transfer function $(S T F)$, the response has a relatively wide, flat all-pass characteristic in the frequency range of interest. Therefore, employing a band-pass $\Sigma \Delta \mathrm{M}$ can result in a high signal bandwidth. For a sinusoidal input signal with $200 \%$ s amplitude at $32 \mathrm{~Hz}$, the simulated output spectrum of the optimized sixth-order continuous-time BP- $\Sigma \Delta \mathrm{M}$ gyroscope is shown in fig. 9(a); the notch denotes the good band-pass characteristics centered at $4.338 \mathrm{kHz}$. However, as shown in 9(b), the non-optimized system gives poorer noise shaping ability. The SNR of the optimized system is $90 \mathrm{~dB}$ within a $64 \mathrm{~Hz}$ bandwidth. For a sampling frequency of $f_{s}=2^{15} \mathrm{~Hz}$, the over-sampling ratio $\left(\mathrm{OSR}=2^{15} \mathrm{~Hz} /[2 \times 64 \mathrm{~Hz})\right)$ is 256 , the noise floor is about $-150 \mathrm{dBV} / \mathrm{Hz}^{1 / 2}$. There is a quadrature error signal at the center frequency originating from fabrication imperfections and thus resulting in an off-axis movement of the proof mass compared to the ideal drive mode axis. This leads to unwanted coupling of the drive into the sense mode. Since the Coriolis signal is very small, the quadrature error signal can dominate the output. A quadrature error signal which due to a $1^{\circ}$ misaligned between actual and ideal drive mode axis results in approximately the same sense mode amplitude as a $200 \%$ s input signal. Nevertheless, as shown in the time-domain simulation results of fig. 10 the RMS displacement of the sense mode was at most 
approximately $10 \mathrm{~nm}$ which is less than $30 \mathrm{~nm}$ as required by the criteria of the GA. The small displacement leads to a significant improvement in linearity of the MEMS gyroscope compared to an open loop interface.

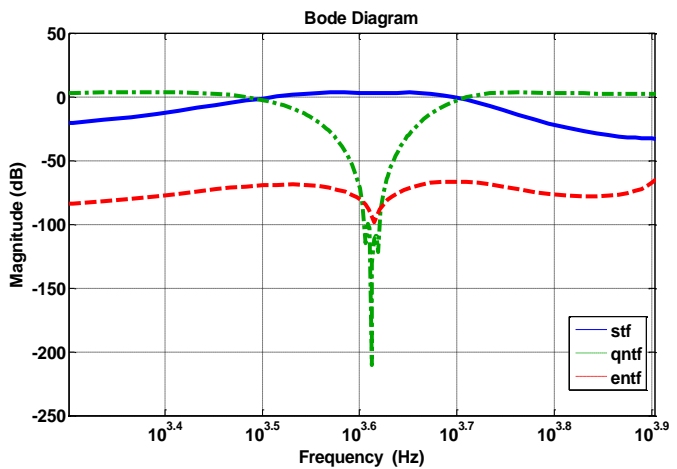

Fig. 8. Magnitude plots of the transfer functions (STF, QNTF, ENTF) for the optimal parameter set obtain by the GA.

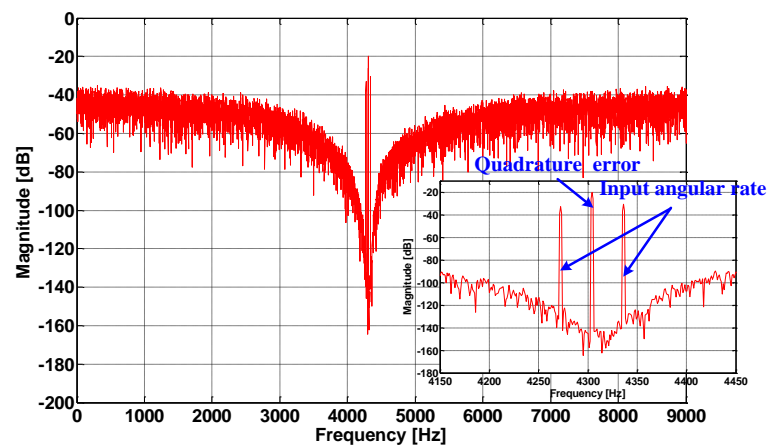

(a)

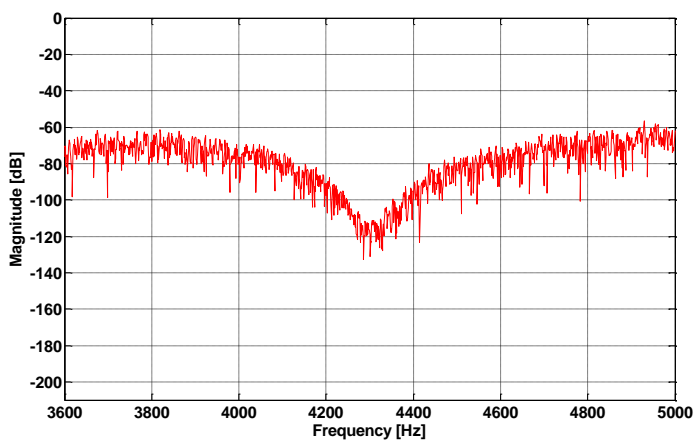

(b)

Fig. 9. (a) Simulated spectrum of the output bitstream of the optimized system for an angular input rate of $200 \% \mathrm{~s}$ at $32 \mathrm{~Hz}$; the inset shows the spectrum around the resonant frequency. (b) The noise floor of the non-optimized system.

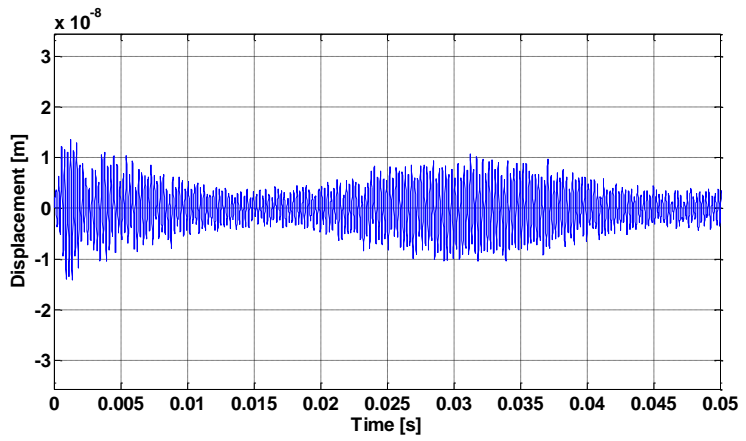

Fig. 10. The sense mode displacement of proof mass for an angular input rate of $200 \%$ at $32 \mathrm{~Hz}$. As required by the criteria of the GA it smaller than $30 \mathrm{~nm}$.

\section{HARDWARE IMPLEMENTATION}

The hardware implementation of the double closed-loop interface follows the functionality of the Simulink model. A block diagram of the system is shown in fig. 11: Three parts can be distinguished: i) the micromechanical gyroscope (in the dark gray region of fig. 11); ii) the drive circuit consisting of the AGC control loop to excite the gyroscope to vibrate at its resonant frequency with constant amplitude and iii) the sixth-order continuous-time BP- $\Sigma \Delta \mathrm{M}$ interface for the sense mode. In order to obtain the final angular rate signal, the output bitstream requires further signal processing, such as phase-sensitive demodulation and low-pass filtering [24]. Fig. 12 shows a scanning electron microscopy (SEM) picture of the $\mathrm{z}$-axis MEMS vibratory gyroscope used in this work. The sensing element, fabricated on a $30 \mu \mathrm{m}$ thick SOI substrate, has a symmetrical design, using a frame structure to mechanically decouple the sense and drive modes [25, 26]. As shown in fig. 13 , in order to investigate the functionality of the double closed-loop control system, the gyroscope packaged under atmospheric pressure, and the circuit implemented by off-the-shelf electronic components were assembled on two four-layer PCBs; each PCB has an overall size of $80 \mathrm{~mm} \times 40 \mathrm{~mm}$, and uses a power supply of $\pm 12 \mathrm{~V}$. In order to reduce electro-magnetic interference on the $\mathrm{PCB}$, the ground plane is split into three parts: gyroscope analog readout circuit ground, AGC control circuit ground and digital circuit ground; they are connected together at a single point. Most circuit blocks were partly reused from previous designs [24]. However, the pick-off circuit, compensator circuit, and resonator circuit require special care; their parameters need to be special adjusted according to the results of the system level optimization.

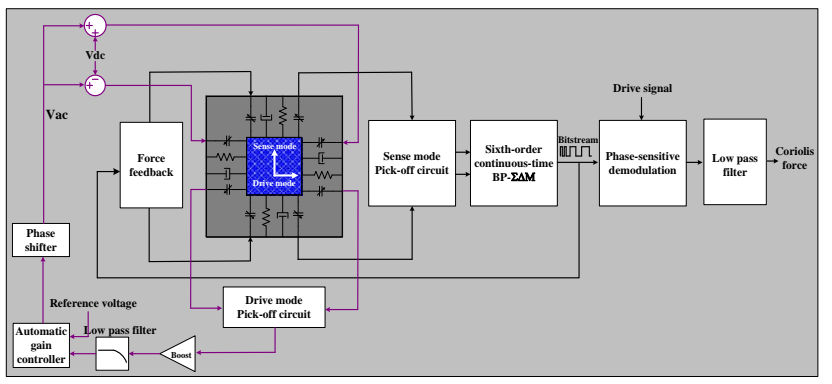

Fig. 11. System-level overview: MEMS vibratory gyroscope (dark grey), interface electronics (light grey).

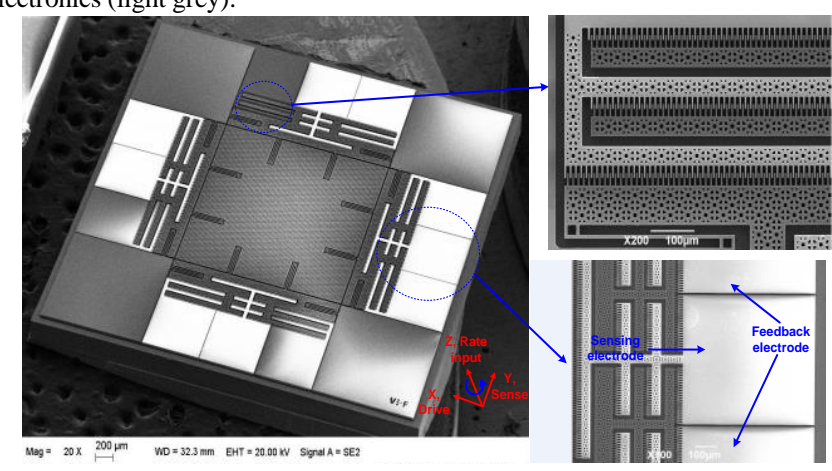

Fig. 12. SEM picture of the symmetrical and fully decoupled SOI gyroscope. The insets show the comb fingers and sensing and feedback electrodes. 


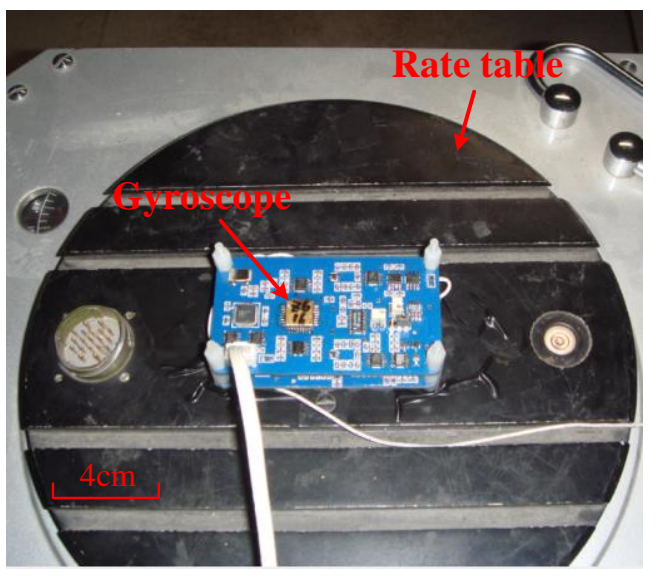

Fig. 13. Photograph of the gyroscope with the double closed-loop control circuit implemented on the PCB. The PCB is shown mounted on a rate table.

\section{A. Pick-off circuit}

The implementation of the pick-off circuit in both control loops is based on a differential capacitance to voltage converter (CVC) for continuous time (CT) circuits, which used an amplitude modulation (AM) technique [31]. As shown in fig. 14 , the topology is fully differential and consists of: a) two frequency-independent half ac-bridges charge integrators, which act as AM modulators, b) two high-pass RC filters, which filter out any signal caused by cross-talk between the feedback and sense capacitors, c) two AM demodulators, and d) an instrumentation amplifier that provides a boost gain.

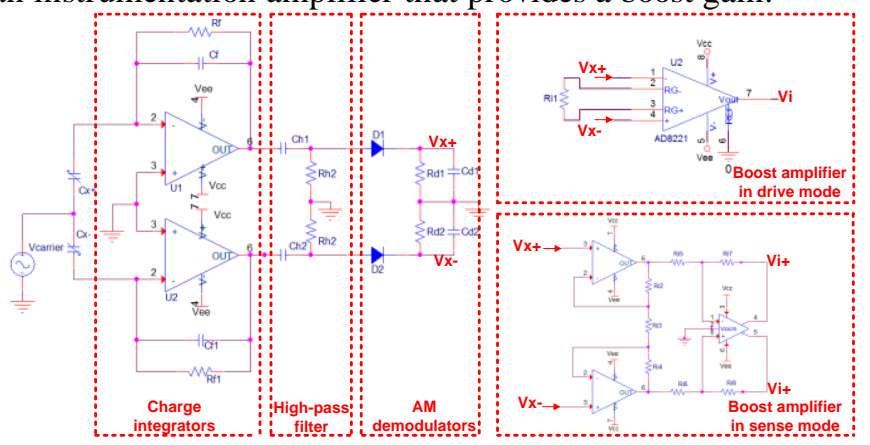

Fig. 14. Schematic diagram of the pick-off circuit

\section{B. Compensator circuit}

The compensator circuit is used to compensate for the phase lag of the sensing element at high frequencies to provide sufficient loop phase margin to ensure stable operation of the sixth-order continuous-time BP- $\Sigma \Delta \mathrm{M}$ stable operation. As shown in fig. 15 , it is implemented with one differential op-amp.

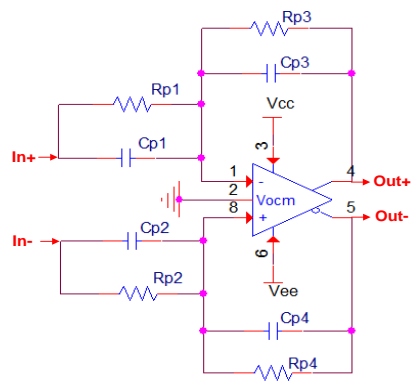

Fig. 15. Schematic diagram of the compensator circuit
The zero frequency $Z_{0}$ and pole frequency $P_{0}$ of the compensator are given (in radians) by

$$
\begin{aligned}
& Z_{0}=\frac{1}{R_{p 1} C_{p 1}}=\frac{1}{R_{p 2} C_{p 2}} \\
& P_{0}=\frac{1}{R_{p 3} C_{p 3}}=\frac{1}{R_{p 4} C_{p 4}}
\end{aligned}
$$

where $C_{p 1}=C_{p 2}=C_{p 3}=C_{p 4}=10 \mathrm{nf}$ was chosen for pragmatic reasons.

According to table III, $Z_{0}=2 \pi \times(4 \mathrm{kHz}), P_{0}=2 \pi \times(194.17 \mathrm{kHz})$, Thus, the values of $R_{p 1}$ and $R_{p 2}, R_{p 3}$ and $R_{p 4}$ are

$$
\begin{aligned}
& R_{p 1}=R_{p 2}=\frac{1}{(2 \pi \times 4000)\left(100 \times 10^{-9}\right)}=3.9 \mathrm{k} \Omega \\
& R_{p 3}=R_{p 4}=\frac{1}{(2 \pi \times 194170)\left(100 \times 10^{-9}\right)}=82 \Omega
\end{aligned}
$$

\section{Resonator circuit}

Two electronic resonators are cascaded with the second order sensing element to from a sixth-order $\Sigma \Delta \mathrm{M}$ loop in the sense mode. Fig. 16 shows the fully differential structure of an active $\mathrm{RC}$ resonator used for the electronic resonators including the HRZ, RZ local feedback paths. The center frequency of resonator $\omega_{r}$ should be equal to the resonant frequency of drive mode (here $\omega_{r}=2 \pi \times 4.304 \mathrm{kHz}$ ), because a resonator is expected to oscillate at its resonance frequency with high gain and exhibit low gain in the rest of the frequency band. The output bitstream controls the HRZ and RZ DACS to realize the multi-feedback topology. The timing diagram shown in fig. 17 illustrates these digital signal derived from the main clock. Using some basic Boolean logic operation, HRZ and RZ DACS can be readily implemented using analog switches which are controlled by the output bitstream and the main clock.

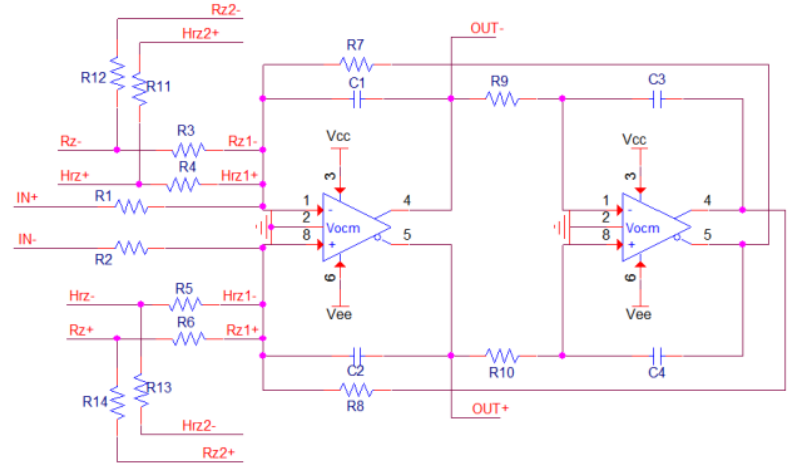

Fig. 16. Schematic diagram of the fully differential $\mathrm{RC}$ resonator with $\mathrm{Hrz}, \mathrm{Rz}$ local feedback paths.

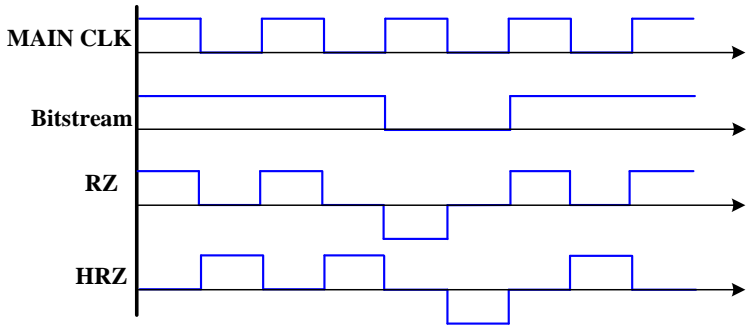

Fig. 17. Timing diagram of Main clock, bitstream, RZ and HRZ. 
$C_{1}, C_{2}, C_{3}$ and $C_{4}$ were chosen as a pragmatic value (here22nf). Thus, $R_{1}$ and $R_{2}, R_{7}$ and $R_{8}, R_{9}$ and $R_{10}$ are all given by

$$
\frac{1}{\omega_{r} \times\left(22 \times 10^{-9}\right)}=1.68 \mathrm{k} \Omega
$$

According to the optimized results presented in table III, $K_{f 1}$, $K_{f 2}, K_{f 3}$ and $K_{f 4}$ is $2.56,1.35,3.72$ and1.07, respectively. Thus $R_{3}$ and $R_{6}, R_{4}$ and $R_{5}, R_{12}$ and $R_{14}, R_{11}$ and $R_{13}$ are given by

$$
\begin{aligned}
& R_{3}=R_{6}=\frac{1.68 \mathrm{k} \Omega}{K_{f 1}}=657 \Omega \\
& R_{4}=R_{5}=\frac{1.68 \mathrm{k} \Omega}{K_{f 2}}=1.24 \mathrm{k} \Omega \\
& R_{12}=R_{14}=\frac{1.68 \mathrm{k} \Omega}{K_{f 3}}=451 \Omega \\
& R_{11}=R_{13}=\frac{1.68 \mathrm{k} \Omega}{K_{f 4}}=1.57 \mathrm{k} \Omega
\end{aligned}
$$

\section{EXPERIMENTAL RESULTS AND DISCUSSION}

The resonant frequencies and the $Q$-factor of the gyroscope drive and sense modes were measured in an open-loop configuration as $4.304 \mathrm{kHz}, 119$, and $4.338 \mathrm{kHz}, 114$, respectively. Referring to table $\mathrm{II}$, as $\theta_{r}$ is $-1.0 \pi$, in order to satisfy the phase angle condition of the closed-loop self-oscillation for the drive mode, the angular phase shift $\theta_{s}$ should be accurately regulated to $-0.5 \pi$. The AGC will dynamically adjust the drive control loop damping, thus a self-oscillation with constant amplitude is sustained. However, the certain uncertainties of the $\theta_{s}$ will lead to a frequency difference $\left(\Delta \omega_{\mathrm{x}}\right)$ between self-oscillation frequency $\left(\omega_{0}\right)$ and natural resonant frequency $\left(\omega_{\mathrm{x}}\right)$ in drive mode. The drive mode displacement $x(t)$ will decrease as $\Delta \omega_{\mathrm{x}}$ increased [5-8], which can deteriorate the Coriolis force.

According to Table II, the DC component of the drive voltage was set to $5 \mathrm{~V}$. The test results show that the gyroscope was excited into resonance along the $\mathrm{x}$ direction with the $\mathrm{AC}$ component set to $8.2 \mathrm{~V}_{\mathrm{p}-\mathrm{p}}$ (peak to peak). Fig. 18 (a) and (b) shows the drift of the amplitude and frequency AC component of the drive signal in a $1 \mathrm{~h}$ long measurement. The measurement results show that the standard deviation of the drive frequency is $0.0136 \mathrm{~Hz}$, with a relative drift of $3.2 \mathrm{ppm}$; the standard deviation of the amplitude is $43.89 \mathrm{mV}$ and the relative drift $10.7 \mathrm{ppm}$. A preliminary test was carried out with zero input rotation under atmospheric pressure. The waveforms of the drive mode self-oscillation and the output bitstream for a zero input angular rate were measured, and are shown in Fig. 19. It can be seen that the output bitstream is a pulse density modulated signal with an approximate mean value of zero, as expected for a zero input signal. Fig. 20 shows the power spectral density (PSD) of the measured output bitstream; there is an obvious band-pass noise shaping form and a deep notch at the gyroscope resonant frequency of 4.338 $\mathrm{kHz}$; the quadrature error appears in the spectrum at a frequency of $4.304 \mathrm{kHz}$, the noise floor is about $-120 \mathrm{dBV} / \mathrm{Hz}^{1 / 2}$ which is higher than in the system level simulation. The discrepancy may be attributed to non-ideal factors, such as noise due to cross-coupling on the PCB, the tolerances of the resistor and capacitor values, fabrication tolerances, excess loop delay and clock jitter $[32,33]$. The noise floor corresponds to $450 \mathrm{nV} / \mathrm{Hz}^{1 / 2}$ which is expected to at the lower limit of what is achievable on a PCB.

For comparative purposes a non-optimized double closed-loop operation mode was implemented simply by randomly altering the system parameter values by $20 \%$ from the GA optimized values. The measured results of the start-up characteristics in non-optimized and optimized drive mode are shown in fig. 21 . It took about $300 \mathrm{~ms}$ and $200 \mathrm{~ms}$ for the gyroscope to self-oscillate to the desired target value without obvious overshoot, respectively. A precision rate table was used to generate the specific rate signal for measurement of the scale factor and the nonlinearity. Fig. 22 shows the measured gyroscope output for different angular input rate signals with a full scale range of $\pm 220 \%$ s (limited by the rate table used in the experiments) in non-optimized and optimized double closed-loop operation modes. The measured scale factors were $17.3 \mathrm{mV} / \%$ s and $22.5 \mathrm{mV} / \% / \mathrm{s}$, respectively. The nonlinearity of MEMS gyroscope for the optimized control system was less than 100ppm. Plots of Allan variance for the gyroscope with the non-optimized and optimized system parameters are shown in fig. 23; it can be seen that the values of zero bias stability is $58^{\circ} / \mathrm{h}$ and $34^{\circ} / \mathrm{h}$, respectively, for $1 \mathrm{~h}$ long measurements. This is mainly due to the sixth-order noise shaping ability of non-optimized and optimized system. The sense mode of gyroscope is cascaded by two resonators with local feedback loops to provide sixth-order noise shaping in the sense mode. The resonator gain $K_{\text {res }}$, the local feedback loop gain $K_{f 1}, K_{f 2}$, $K_{f 3}, K_{f 4}$ and feedback voltage $V_{f}$ all have significant influence on the noise shaping ability of the system. As shown in fig. 9(a) and (b), the optimized system has better sixth-order noise shaping resulting in better zero bias stability. Fig. 24 shows the frequency response of the non-optimized and optimized system is about $98 \mathrm{~Hz}$ and $110 \mathrm{~Hz}$, respectively.

Most high performance MEMS gyroscopes described in the literature [34-36] use vacuum packaging, resulting in Q-factors $>1000$ but therefore requiring active resonant mode matching control. In [34], a $60 \mu \mathrm{m}$ thick tuning fork gyroscope with ASIC electronic circuit, was packaged at high-vacuum achieving a quality factor of 36,000 . The zero bias stability was $0.16^{\circ} / \mathrm{h}$ with active resonant mode-matching control. The scale factor and noise floor was $88 \mathrm{mV} / \%$ and $15 \mathrm{uV} / \mathrm{Hz}^{1 / 2}$, respectively. However, the maximum achievable bandwidth was only $10 \mathrm{~Hz}$. The high performance symmetrical MEMS gyroscope packaged at atmospheric pressure was also reported in $[37,38]$, the lowest noise floor is $2.5 \mathrm{uV} / \mathrm{Hz}^{1 / 2}$. The bandwidth was limited within $50 \mathrm{~Hz}$. The scale factor and zero bias stability can achieve to $15.2 \mathrm{mV} / \%$ s and $60 \% / \mathrm{h}$, respectively. In this paper, the gyroscope was packaged at atmospheric pressure. The quality factor of drive and sense modes were only 119 and 114 , respectively. The zero bias stability was $34^{\circ} / \mathrm{h}$, the scale factor and noise floor were $22.5 \mathrm{mV} / \%$ and $450 \mathrm{nV} / \mathrm{Hz}^{1 / 2}$, respectively. One benefit is a relatively high bandwidth, which was $110 \mathrm{~Hz}$ under closed loop control. The performance of the developed sensor system can be extended further by using an 
ASIC interface circuit and re-designing the sensing element to increase the quality factor of gyroscope.

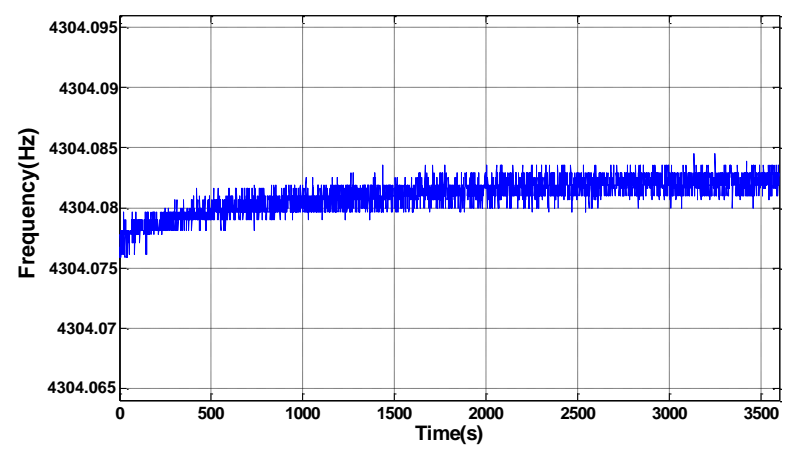

(a)

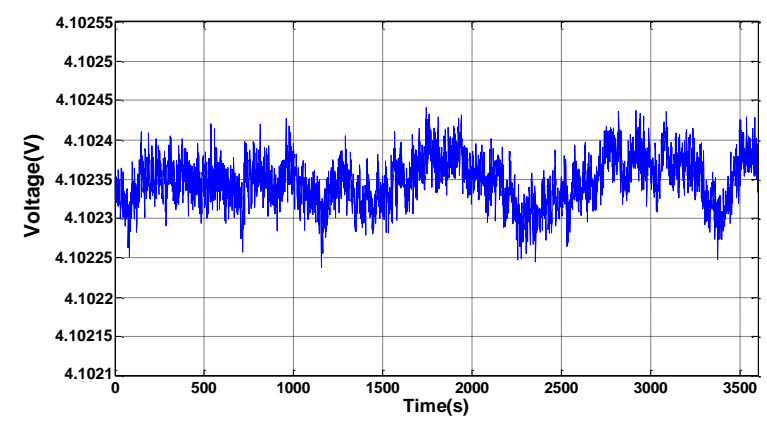

(b)

Fig. 18. Measurement of frequency (a) and amplitude (b) drift of the AC driving sinusoidal signal for 1 hour in optimimized AGC drive closed loop.

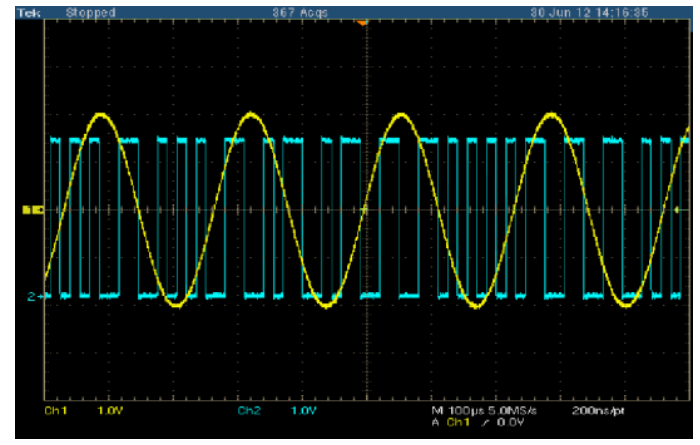

Fig. 19. The output bitstream (blue) for a zero input angular rate signal and the sinusoidal drive mode oscillation (yellow).
○ X:4.304 kHz
口X: $4.24 \mathrm{kHz}$
Y:-93.472 dBV/rtHz
$\mathrm{Y}:-127.484 \mathrm{dBV} / \mathrm{tH} \mathrm{Hz}$

Pwr Spec 1

$-50$

$\mathrm{dBV} / \mathrm{rtHz}$

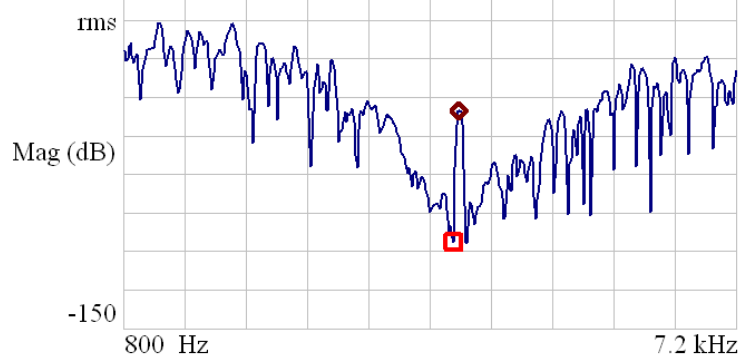

Fig. 20. Measured spectrum of the output bitstream with a zero angular rate input.

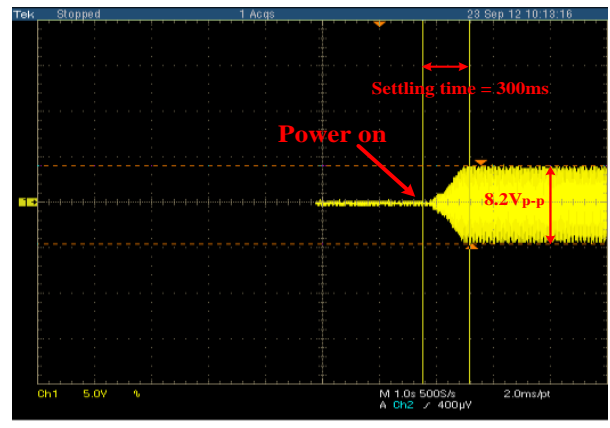

(a)

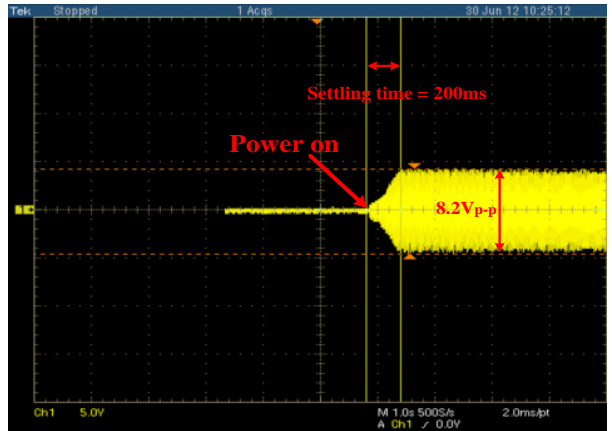

(b)

Fig. 21. The settling time of the self-oscillation in non-optimized (a) and optimized (b) drive mode is $300 \mathrm{~ms}$ and $200 \mathrm{~ms}$, respectively.

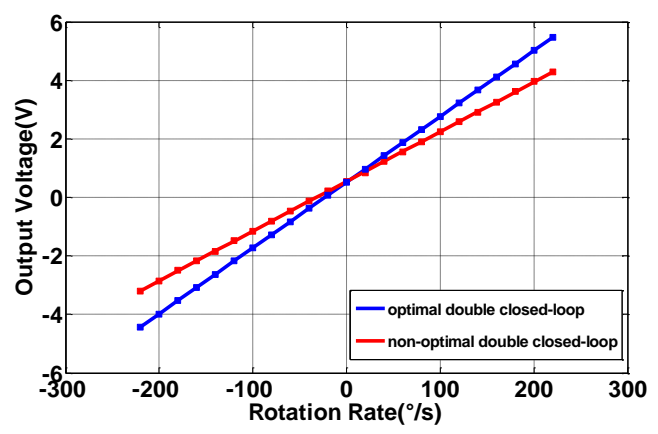

Fig. 22. Measured output signals of the gyroscope in optimized and non-optimimized double closed- loop (DC angular rate inputs in a range of \pm $220 \% \mathrm{~s}$ ). The scale factor and nonlinearity of the gyroscope controlled by the optimized double closed-loop interface are $22.5 \mathrm{mV} / \%$ s, <100ppm, respectively. This compares to the non-optimized control system for which the corresponding values are $17.3 \mathrm{mV} / \%$ s and $200 \mathrm{ppm}$.

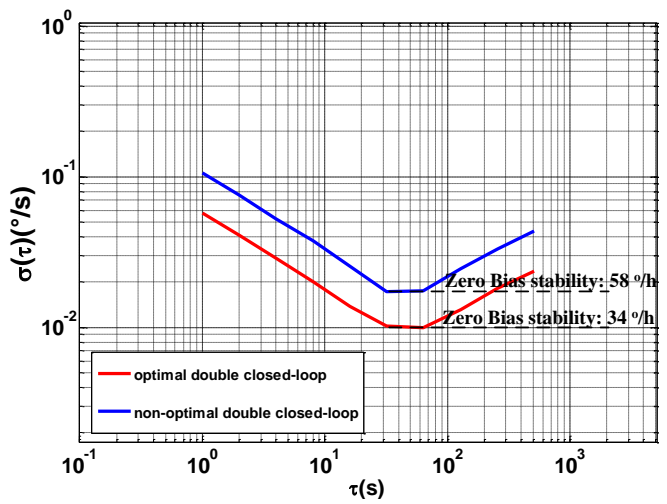

Fig. 23. Plots of Allan variance of the gyroscope in optimized and non-optimized double closed-loop control system. 


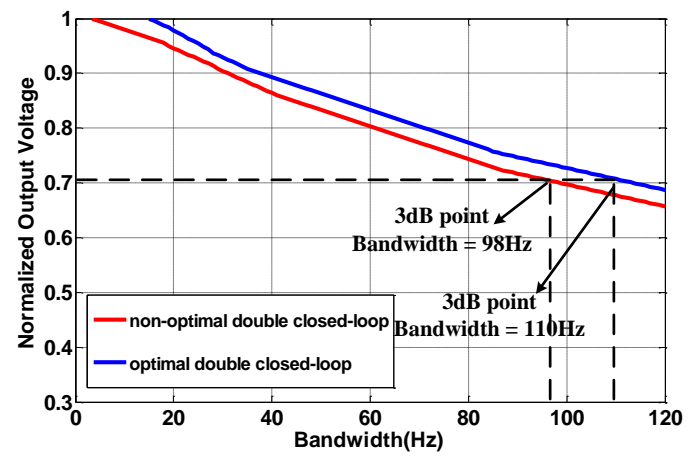

Fig. 24. Frequency response of the gyroscope measured up to $120 \mathrm{~Hz}$.

Table IV summarizes a comparison of the overall measured performance of the optimized double-closed loop gyroscope system and a non-optimized operation mode. It can be seen that the use of the proposed GA optimization method, the performance of the MEMS gyroscope system is considerably improved.

To quantify the power consumption saving due to the low sampling frequency made possibly by the band-pass $\Sigma \Delta \mathrm{M}$ sense mode interface, we increased the sampling frequency from $32 \mathrm{kHz}$ (as used in the presented test results) to $2 \mathrm{MHz}$. The power dissipation increased from $3.6 \mathrm{~W}$ to $6.72 \mathrm{~W}$. The figures should be seen to only demonstrate a trend, not absolute, since no particular attention to power consumption was paid when designing the interface circuit; furthermore an ASIC implementation would reduce the power consumption by an order of magnitude.

TABLE IV

COMPARISON OF OPTIMIZED AND NON-OPTIMIZED GYROSCOPE SYSTEM OPERATING AT ATMOSPHERIC PRESSURE

\begin{tabular}{ccc}
\hline \hline Performance parameters & Optimized & Non-optimized \\
\hline$Q_{x}$ & 119 & 119 \\
$Q_{y}$ & 114 & 114 \\
Scale factor $(\mathrm{mV} / \%$ s $)$ & 22.5 & 17.3 \\
Zero bias stability $\left({ }^{\circ} / \mathrm{h}\right)$ & 34 & 58 \\
Nonlinearity $(\mathrm{ppm})$ & $<100$ & 200 \\
Bandwidth $(\mathrm{Hz})$ & 110 & 98 \\
Settling time $(\mathrm{ms})$ & 200 & 300 \\
Noise floor $(\mathrm{dBV} / \mathrm{Hz}$ & -90 \\
\hline \hline
\end{tabular}

\section{CONCLUSION}

This paper presents the design and implementation of an optimized double closed-loop control system for a MEMS vibratory gyroscope (AGC self-oscillation for the drive mode, sixth-order continuous-time BP- $\Sigma \Delta \mathrm{M}$ force feedback control system for the sense mode). The corresponding Simulink mode is established and optimized by a genetic algorithm (GA). In order to prove the functionality of optimized system, a symmetrical and fully decoupled MEMS gyroscope was designed and fabricated in a SOI process. The system was implemented in hardware using standard off-the-shelf electronic components. The PSD of the measured output bitstream reveals an obvious band-pass noise shaping form at the sense resonant frequency, the noise floor is approximately $-120 \mathrm{dBV} / \mathrm{Hz}^{1 / 2}$. The gyroscope system was tested on a rate table, the test results show that the optimized double closed-loop control system considerably improves the setting time, scale factor, zero bias stability, bandwidth of the MEMS vibratory gyroscope when compared to a non-optimized operation.

\section{ACKNOWLEDGMENT}

The authors wish to thank Dr Reuben Wilcock for useful discussions on the GA optimization.

\section{REFERENCES}

[1] N. Yazdi, F. Ayazi, K. Najafi, "Micromachined inertial sensors," Proc. IEEE, vol.86, pp.1640-1659, 1998.

[2] H. Xie, G. Feder, Integrated microelectromechanical gyroscopes, J. Aerosp. Eng., vol.16, pp. 65-75, 2003.

[3] R. Neul, et al., Micromachined angular rate sensors for automotive application, IEEE J. sensors, vol.7, pp.302-309, 2007.

[4] S. H. Kim, J. Y. Lee, C. H. Kim and Y. K. Kim, A bulk-micromachined single crystal silicon gyroscope operating at atmospheric pressure, in proc. Transducers' 01 , Munich, Germany, 2001, pp. 476-479.

[5] J. Cui, X. Z. Chi, H. T. Ding, L. T. Lin, Z. C. Yang, G. Z. Yan, Transient response and stability of the AGC-PI closed-loop controlled MEMS vibratory gyroscopes, J. Micromech.Microeng. vol.19, pp. 1-17. 2009.

[6] B. Eminoglu, S.E. Alper, T. Akin, An optimized analog drive-mode controller for vibratory MEMS gyroscope, Proc. Eurosensors XXV, Athens, Greece, 2011, pp. 1309-1312.

[7] R. Oboe, R. Antonello, E. Lasalandra, G. S. Durante, L. Prandi, Control of a Z-axis MEMS vibrational gyroscope, IEEE/ASME Trans. Mechatron., vol.10, pp. 364-370, 2005.

[8] B. Yang, B. Zhou and S. Wang, A precision closed-loop driving scheme of silicon micromachined vibratory gyroscope, J. Phys.: Conf. Ser., vol.34, pp. 57-64. 2006.

[9] R. T. M'Closkey and A. Vakakis, Analysis of a microsensor automatic gain control loop, in proc. American Control Conf., San Diego, USA, 1999, pp. 3307-3311.

[10] R. T. M'Closkey, A. Vakakis and R. Gutierrez, Mode localization induced by a nonlinear control loop, Nonlinear Dyn., vol.3, pp.221-236, 2001.

[11] Y. C. Chen, R. T. M'Closkey, T. A. Tran, B. Blaes, A control and signal processing integrated circuit for the JPL-Boeing micromachined gyroscopes, IEEE trans. Control Syst. Technol, vol.13, pp.286-300, 2005.

[12] D. J. Kim, R. T. M'Closkey, Real-time tuning of JPL-Boeing MEMS gyro dynamics, American control conf., Portland, USA, 2005, pp.3598-3603.

[13] W. T. Sung, S. Sung, J. Y. Lee, T. Kang, Y. J. Lee and J. G. Lee, Development of a lateral velocity-controlled MEMS vibratory gyroscope and its performance test, J. Micromech.Microeng. vol.18, pp. 1-17. 2008.

[14] V. P. Petkov, B. E. Boser, A fourth-order $\Sigma \Delta \mathrm{M}$ interface for micromachined inertial sensors, IEEE J. Solid-State circuits, vol.40, pp.1602-1609, 2005.

[15] V. P. Petkov, B. E. Boser, High-order electromechanical $\Sigma \Delta \mathrm{M}$ modulation in micromachined inertial sensors, IEEE Trans. Circuits Syst.-I, vol.53, no.5, pp.1016-1022, 2006.

[16] J. Raman, E. Cretu, P. Rombouts, L. Weyten, A digitally controlled MEMS gyroscope with unconstrained sigma-delta force-feedback architecture, in Proc. $19^{\text {th }}$ IEEE Int. Conf. Micro Electro Mech. Syst., Istanbul, Turkey, 2006, pp. 710-713.

[17] J. Raman, E. Cretu, P. Rombouts, L. Weyten, A closed-loop digitally controlled MEMS gyroscope with unconstrained sigma-delta force-feedback, IEEE J. sensors, vol.9, pp. 297-305, 2009.

[18] H. Rodjegard, D. Sandstrom, P. Pelin, N. Hedenstierna, D. Eckerbert, G. I. Andersson, A digitally controlled MEMS gyroscope with $3.2 \mathrm{deg} / \mathrm{Hr}$ stability, $13^{\text {th }}$ Conf. on Transducers' 05 , Seoul, Korea, 2005, pp. 535-538.

[19] Y. Dong, M. Kraft, W. Redman-White, High order band-pass sigma delta interfaces for vibratory gyroscopes, in Proc. $4^{\text {th }}$ IEEE Conf. Sensors, Irvine, USA, 2005, pp. 1080-1083.

[20] Y. Dong, M. Kraft, W. Redman-White, Micromachined Vibratory gyroscopes controlled by a high-order band-pass sigma-delta modulator, IEEE J. sensors, vol.7, pp. 59-69, 2007.

[21] Y. Dong, M. Kraft, W. Redman-White, 2007 High order noise shaping filters for high performance inertial sensors IEEE Trans. Instrum. Measurement, vol.56, pp. 1666-1674, 2007. 
[22] H. Ding, Z. Yang, G. Yan, M. Kraft, R. Wilcock, MEMS gyroscope control system using a band-pass continuous-time sigma-delta modulator, $9^{\text {th }}$ IEEE Conf. Sensors, Hawaii, USA, 2010, pp. 868-872.

[23] J. Luo, H. Ding, M. Kraft, A new design methodology for electro-mechanical sigma-delta modulators, Proc. $4^{\text {th }}$ IEEE Int. Conf. on Nano/Micro Engineered and molecular System, Shenzhen, China, 2009, pp. 881-884.

[24] F. Chen, H. Chang, W. Yuan, R. Wilcock, M. Kraft, Parameter optimization for a high-order band-pass continuous-time sigma-delta modulator MEMS gyroscope using a genetic algorithm approach, J. Micromech. Microeng, vol.22 (105006), 2012.

[25] H. Chang, Y. Zhang, J. Xie, Z. Zhou, W. Yuan, Integrated behavior simulation and verification for a MEMS vibratory gyroscope using parametric model order reduction, J. Microelectromech. Syst., vol.19, pp. 282-293, 2010.

[26] H. Chang, J. Xie, Q. Fu, Q Shen, W. Yuan, Micromachined inertial measurement unit fabricated by a SOI process with selective roughening under structures, Micro \& Nano letter, vol.6, pp.486-489, 2011.

[27] R. Maurino, P.Mole, A $200 \mathrm{MHz}$ IF 11 bit fourth-order bandpass sigma-delta ADC in SiGe, IEEE J.Solid state Circuits, vol.35, pp.959-967, 2000

[28] J. Lota, M. Al-Janabi, I. Kale, Nonlinear-stability analysis of higher order D-S modulators for DC and sinusoidal inputs, IEEE Trans. Instrum. Meas. Vol.57, pp.530-542, 2008.

[29] Y. Dong, M. Kraft, W. Redman-White, Force feedback linearization for higher-order electromechanical sigma-delta modulators. J. Micromech. Microeng., vol. 16, pp.54-60, 2006.

[30] R. Wilcock, M. Kraft, Genetic algorithm for the design of electro-mechanical Sigma Delta Modulator MEMS Sensors, MDPI J. Sensors, vol.10, pp.9217-9238, 2011.

[31] J.C. Lotters, W. Olthuis, P.H. Veltink, P. Bergveld, A sensitive differential capacitance to voltage converter for sensor applications. IEEE Trans. Instrum. Meas. Vol.48, pp.89-96, 1999.

[32] J.A. Cherry, W.M. Snelgrove, Excess loop delay in continuous-time delta-sigma modulators, IEEE Trans.Circuits Systems II:Analog Digital Signal Process. Vol.46, pp.376-389, 1999.

[33] J.A. Cherry, W.M. Snelgrove, Clock jitter and quantizer metastability in continuous-time delta-sigma modulators, IEEE Trans.Circuits Systems II:Analog Digital Signal Process. Vol.46, pp.661-676, 1999.

[34] A. Sharma, M. F. Zaman, F. Ayazi, A sub-0.2\% $/ \mathrm{hr}$ bias drift micromechanical silicon gyroscope with automatic CMOS mode-matching, IEEE J. Solid-State Circuits, vol.44, pp.1593-1608, 2009.

[35] S. E. Alper, Y. Temiz, T. Akin, A compact angular rate sensor system using a fully decoupled silicon-on-glass MEMS gyroscope, J. Microelectromech. Syst.,vol.17, pp. 1418-1429, 2008.

[36] S. Sonmezoglu, S. E. Alper, T. Akin, An automatically mode-matched MEMS gyroscope with $50 \mathrm{hz}$ bandwidth, $25^{\text {th }}$ IEEE Int. Conf. Micro Electro Mech. Syst., Paris, France, 2012, pp. 523-526.

[37] S.E. Alper, K. Azgin, T. Akin, A high-performance silicon-on-insulator MEMS gyroscope operating at atmospheric pressure, Sensors Actuators A, vol. 135, pp.34-42, 2007.

[38] H. Ding, X. Lin, L. Lin, X. Chi, J. Cui, M. Kraft, Z. Yang, G. Yan, A high-resolution silicon-on-glass $\mathrm{Z}$ axis gyroscope operating at atmospheric press, IEEE Sensors J., vol.10, pp.1066-1074, 2010.

Fang Chen received the B.S. and M.S. degree in mechanical and electronic engineering from Northwestern Polytechnical University, Xi'an, China, in 2006 and 2009 , respectively. He is working toward the Ph.D. degree at the Key laboratory of Micro/Nano Systems for Aerospace, Northwestern Polytechnical University, Ministry of Education, Xi'an, China.

His research interests are the design of electronics and control strategies for MEMS inertial sensors.

Weizheng Yuan received the Ph.D. degree in mechanical engineering from Northwestern Polytechnical University, Xi'an, China, in 1996.

$\mathrm{He}$ is currently the director of the Key laboratory of Micro/Nano Systems for Aerospace, Northwestern Polytechnical University, Ministry of Education. His research interests include microsensors, microoptics, and applications of microelectromechanical systems.

Honglong Chang received the B.S., M.S., and Ph.D. degrees in mechanical engineering from Northwestern Polytechnical University (NPU), Xi' an, China, in 1999, 2002, and 2005, respectively. He is currently a Professor with the Key laboratory of Micro/Nano Systems for Aerospace, Northwestern Polytechnical University, Ministry of Education, where he is also currently the Dean of the Department of Microsystem Engineering. From Oct.2011 to Nov. 2012, he was a
Visiting Associate (Faculty) with the Micromachining Laboratory, California Institute of Technology, Pasadena. His research interests include microelectromechanical systems (MEMS) design tools, MEMS inertial sensors, and bio-MEMS. He has published more than 17 international journal papers and more than 20 international conference papers in MEMS field.

Guangmin Yuan received the M.S. degree in mechanical engineering from Northwestern Polytechnical University, Xi'an, China, in 2007.

$\mathrm{He}$ is now working as an engineer at the Key laboratory of Micro/Nano Systems for Aerospace, Northwestern Polytechnical University, Ministry of Education. His current research interest focuses on the design of readout circuits for MEMS sensors.

Jianbing Xie received the Ph.D. degree in mechanical engineering from Northwestern Polytechnical University, Xi'an, China, in 2010.

$\mathrm{He}$ is now working as a Faculty Member at the Key laboratory of Micro/Nano Systems for Aerospace, Northwestern Polytechnical University, Ministry of Education. His current research interests focuses on the design and fabrication of microgyroscopes and microaccelerometers.

Michael Kraft received the Dipl.-Ing.(Univ.) degree in electrical and electronics engineering from the Friedrich Alexander Universität Erlangen-Nürnberg, Erlangen, Germany, in 1993 and the Ph.D. degree from Coventry University, Coventry, U.K., in 1997.

Since Oct. 2012 he is a Professor of Integrated Nano- and Microsystems with the Faculty of Engineering Sciences at the University of Duisburg-Essen Germany. $\mathrm{He}$ concurrently heads the Department of Micro- and Nanosensors at the Fraunhofer Institute for Microelectronic Circuits and Systems in Duisburg. Before, he spent 13 years as an academic at the School of Electronics and Computer Science, University of Southampton, Southampton, U.K, where he also acted as the Director of the Southampton Nanofabrication Centre. He also spent two years at the Berkeley Sensors and Actuator Centre, University of California, Berkeley, working on integrated MEMS gyroscopes. In his career he has focused on novel micro- and nanofabrication techniques, microsensors, and actuators and their interface circuits, particularly for capacitive sensors. He has a broad interest in MEMS and nanotechnology ranging from process development to system integration of MEMS and nanodevices. He has published over 160 peer-reviewed journal and conference papers as an author or coauthor. He also contributed to three text books on MEMS and edited a book on MEMS for aerospace and automotive applications.

Dr. Kraft currently serves on several steering and technical committees of international conferences such as IEEE Sensors, Eurosensors, and the Micromechanics and Microsystems Europe Workshop. 